\title{
Exploring Interaction Strategies in the Context of Sleep
}

\author{
Dzmitry Aliakseyeu $^{1}$, Jia Du ${ }^{1}$, Elly Zwartkruis-Pelgrim ${ }^{1}$, and Sriram Subramanian ${ }^{2}$ \\ ${ }^{1}$ Philips Research Europe, 5656 Eindhoven, The Netherlands \\ ${ }^{2}$ Computer Science, University of Bristol, U.K. \\ \{dzmitry.aliskseeyeu, jia.du, \\ elly.zwartkruis-pelgrim\} @philips.com, \\ sriram@cs.bris.ac.uk
}

\begin{abstract}
This paper highlights opportunities and considerations when developing interaction techniques in the relatively unexplored area of sleep. We do this by first describing the sociological aspects of sleep such as the need for scheduling and coordinating sleep within a collective, followed by a description of the physiological aspects such as the circadian rhythm and sleep stages. We then examine how some external factors like the location of sleep and family settings can affect sleep to highlight potential design opportunities. We finish the paper by describing five distinct themes such as scheduling for a collective, supporting sleep transitions and feigning and inhibiting sleep around which design opportunities are explored. The main contribution of this paper is a discussion on the phenomenon of sleep and its position in the social life to provide a rich understanding of sleep and a set of opportunities for interaction design research around sleep.
\end{abstract}

Keywords: Sleep, interaction design, sleep quality, bedroom context.

\section{Introduction}

Sleep is a fundamental and pervasive behavior among members of the animal kingdom. An average human needs about 7 to 8 hours of sleep a night [28]. Sleep affects our physical and mental health and is essential for normal functioning of all processes within our body. For example, sleep has a major restorative function for the human body and plays an essential role in memory processes and learning.

Although there isn't a clear understanding of the fundamental purpose of sleep from a neuroscience or biology perspective, the functional necessity of sleep is demonstrated by showing that failing to do so for even a few days leads to a broad repertoire of pathological outcomes [43]. Lack of sleep can lead to a decrease in metabolic activity of the brain, a weakened immune system, decreased alertness and ability to focus. Sleep reduction is also linked to weight gain [40], increased risks of breast cancer [54] and impaired glucose intolerance [42]. It is therefore essential that humans get the right amount of sleep.

However, a busy lifestyle and work pressures have led to an increase in waking hours at the expense of sleeping hours. In the 2007 US National Sleep Foundation Survey, about $65 \%$ of the people stated having some kind of sleep problem and $44 \%$ 
reported sleep problems almost every night resulting in symptoms close to insomnia [36]. The pharmacological market for sleep disorders was valued at \$7bn in 2008 and growing.

Sleep has gained increased awareness not only because of its widely recognized functional significance in health but also its influence in people's social life and performance. We may look at sleep as an activity for resting the brain and body, but we can also look at sleep as a special stage, e.g. bedtime ritual, where we interact with the world and people around us in a unique style.

Experiences around sleep are an interesting area that has been dominated by the bedding industry for years. Only recently has one seen the emergence of solutions in the bedroom that focuses on creating new experiences around people's sleep. For example, products for directly improving sleep or sleep transitions include devices such as Ecotones Sound Therapy Machine ${ }^{(}$and Philips' Wake-up Light ${ }^{\circledR}$. They improve sleep by reducing the environmental disturbances (like sound) and help people to fall asleep by playing relaxing sounds, creating a dusk lighting ambiance or waking people up by gradually increasing light.

While addressing specific sleep related problems through sensing and interaction technology is important, we argue that sleep should be considered as a holistic part of our lifestyle. Understanding the social, psychological, and physiological aspects of sleep can lead to design opportunities that not only lead to improved quality of sleep but also an improved quality of life.

In this paper we examine the various design opportunities that exist in the context of sleep. We start by reviewing HCI literature that is related to sleep followed by a review the physiological, psychological and sociological aspects of sleep. We finally present various design opportunities that arise from clever integration of the factors with socio-physiological aspects.

The paper has three main contributions:

- Through a review of the literature we describe the phenomenon of sleep and its position in the social life to provide a rich understanding of it.

- Expand the design space of interactive technologies to include sleep by describing factors that need to be considered when designing for sleep.

- Identify five sleep related themes that have high potential and need for interaction design.

\section{Sleep and HCI}

Research into interactive technologies in the home is growing in popularity. There are numerous research articles on all aspects of the interconnected living room and kitchen yet there is very little research into HCI for the bedroom. This is despite the fact that on average a person spends most of their time in their bedroom when at home; for most of this time they will be asleep. This limited research interest in the past is perhaps a consequence of considering sleep as a state of inactivity. In this section we review sleep monitoring products and fairly limited amount of HCI literature that looks at interactive solutions to support sleep. 


\subsection{Sleep Monitoring Tools}

There are a number of technologies available for monitoring the sleep. Polysomnography (PSG) [34], is often used in clinical settings and allows researchers to view physiological status of a person during sleep e.g. eye movements, chin muscle activity, brain activities and heart rate. It is regarded as the gold standard to measure sleep and wake activities [23]. However, current PSG measurement requires attaching many physiological sensors on the body, which cannot be done unobtrusively [12] and therefore less suited for home use. Actigraphy [7] is commonly used as an unobtrusive alternative to PSG. Actigraphy is a watch like device that is worn on one's arm, and measures movements with an accelerometer and interprets sleep and wake patterns accordingly $[19,46]$. It has been shown to be valid in assessing sleep and wake activity but is less accurate for monitoring detailed sleep information like sleep stages and sleep onset [1]. Actigraphy is commonly used together with subjective sleep assessment methods such as a sleep diary [34], especially in longitudinal studies in clinical settings. Many commercial products also incorporated the concept of actigraphy. One such example is the iPhone application EasyWakeup (easywakeup.net) which, when placed underneath the pillow, can detect the movements of a person and it will wake up a person during a relatively lighter sleep phase within a user defined wake up time window.

Zeo [58] is a new sleep monitoring device that is claimed to be able to monitor single PSG by three sensors integrated in a headband. Zeo can provide more accurate information about sleep quality, quantity and sleep staging than an actiwatch. It is also less obtrusive than full PSG measurement.

\subsection{Interaction Design around Sleep}

Most of the research in HCI and design involving sleep has revolved around the alarm clock. Landry et al. [27] extended a standard alarm clock to include contextual information (traffic, weather, early meeting, etc.) to inform the user of any unusual situations that would require an adjustment in the usual morning routine. Schmidt [48] has developed a networked alarm clock that is connected with the social network of a user. The user can define when to be awoken by including conditions such as 'wake me if persons A \& B are already out of the bed', i.e. the wake up time (alarm) can be defined by the status of other members of the network. The reverse alarm clock designed by Ozenc et al. [39] encourages children to stay in bed and avoids disturbing their parents during the night or too early in the morning. Choe et al. [8] carried out interviews and surveys to synthesize concepts for supporting healthy sleep behavior such as Sleep monitoring clock and role playing game to promote healthy sleep.

Kim et al. [24] developed a network enabled alarm clock so people can share their sleeping status within a social group. They found that sharing sleeping patterns allowed participants to feel more connected and intimate [24]. Sleep therefore can become an important part of a co-presence system that contributes to achieving a higher level social connectedness. Other examples of systems that use the bed for connecting remote people to improve intimacy of couples sleeping in different locations are The Bed by Dodge [11], Telematic Dreaming by Sermon [50] and Somnia [47]. Each of these projects connects sleepers in two different locations 
through sensory immersions such as warming the pillow and transmitting audio and video.

Various creative solutions have been proposed for this unique location and its accompanied user state. However, using existing HCI standards is not sufficient to meet future user needs and expectations. A good understanding of the specific context and user state are needed first in order to come to HCI standards for this specific area. In the following chapters we will present different aspects that can be considered in this respect.

\section{Sleep}

Humans spend on average about a third of their life sleeping. Although historically sleep has been considered to be a state of inactivity, scientists now believe that sleep is a dynamic process with brain activity.

Sleep is the most important form of periodic remission which requires scheduling, coordination and careful consideration for ones vulnerability [57]. These activities of sleep are of social significance and present rich opportunities for exploring design solutions. Most of the social aspects of sleep are often dictated by the human sleep physiology and brain activity. For example, bed time rituals of transitioning from being awake to asleep and vice-versa have emerged from our intuitive understanding of the physiology of sleep. Important differences in sleeping practices in different countries (monophasic sleep vs. the siesta culture) can be better understood by looking at the human biological clock.

To identify what role interactive technologies can play in supporting sleep and activities around sleep we first need to understand what role sleep plays in our life and what sociological, cognitive and physiological processes define it. In this section we give a very brief overview of relevant work on the physiology, sociology and psychology of sleep.

\subsection{Human Sleep from a Physiological Perspective}

The sleep and wake cycles are driven by the circadian clock and homeostatic sleep drive.

Circadian Clock. The internal circadian clock regulates rhythmic variations in physiological and behavioral processes, such as core body temperature, food intake and sleep [41]. The rhythm of this circadian clock is depicted in figure 1. The circadian clock potentiates wakefulness (and alertness) at one phase of the diurnal cycle, while facilitating sleep and its attendant processes at the opposite phase [44]. Engaging in behaviors that are not synchronized with the circadian clock, such as travelling through different time zones or shift work, will result in a misalignment between the external environment and the circadian clock.

This internal clock naturally maintains a periodic rhythm of slightly more than 24 hours. External factors, most importantly light, provide daily resets of this internal mechanism to a 24 hour cycle. Studies have shown that bright light administered during the early biological night will delay the timing of the circadian system, whereas bright light administered during the late biological night will advance the 
timing of the circadian system [22]. Exposure to light suppresses the release of melatonin, a hormone that promotes sleep.

Sleep Onset. Sleep onset refers to the transition from wakefulness to sleep. The period is defined as the time spent after a person goes to bed and the light is turned off to entering stage one sleep. This process is usually observable via brain signal changes $[14,16]$. Transition from sleep onset to light sleep has been described as the process of gradual reduction of arousal through thalamic deactivation, a disappearance of saccades, a reduction in endogenous blinking and an appearance of slow eye movements. During sleep onset, humans still maintain their reaction to external stimuli, even till stage 1 and 2 sleep, though responses and reaction time decrease slowly as we enter deep sleep [37].

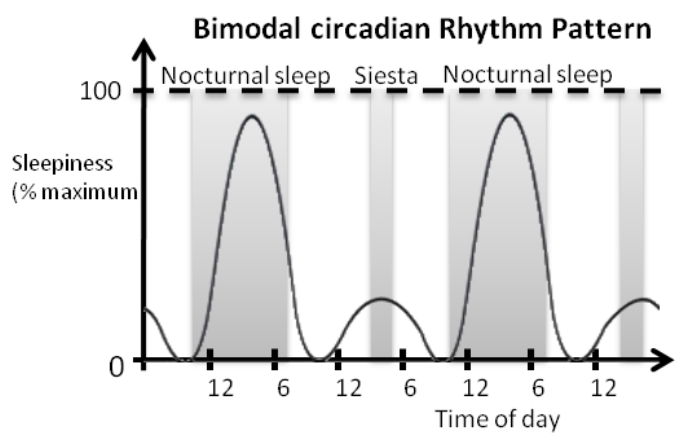

Fig. 1. The biological clock drives a person's sleep and wake pattern throughout a day, being most sleepy in the early morning and second in the afternoon. Based on [31].

Waking Up. The process of awakening is accompanied with reactivation of consciousness and alertness. Opposite to sleep onset, increased core body temperature and heart rate corresponds to the reduced subjective sleepiness are observed [2]. When awakened abruptly from sleep, for example by a sudden noise, one may feel drowsy and find it difficult to remain awake. This is known as sleep inertia, and may last for minutes up to several hours [25] (sleep inertia and accompanied feeling will also depend on factors such as sleep stage people are awakened from, the amount of time they have slept, their circadian phase and so on). So it is essential that a person does not wake up abruptly from sleep but that the wake up process is gradual.

Sleep Drive. Just as a person needs to drink when losing fluid during exercising, a person needs to sleep when she has not slept enough. The drive for sleep and the need for drinking are regulated by the human's internal mechanism known as homeostatic drive [31]. In terms of sleep, homeostatic drive not only takes into account the hours a person has been active during the day but also how many hours were lacking from the person's previous nights. Therefore he/she may feel sleepier when homeostatic sleep drive functions as a result of an insufficient amount of sleep.

Sleep Stages. Human sleep is not a binary switch which is ON or OFF but goes through stages and cycles, alternating between light and deep sleep stages. Scientists have characterized those stages as Rapid Eye Movement (REM) sleep and Non-REM 
sleep. Normal nocturnal sleep consists of four or five sleep cycles [20] each including several stages of Non-REM sleep and REM sleep. A complete sleep cycle includes progression from stage one to stage four sleep and back to stage one and then entering REM sleep, after which a new cycle starts again, each lasting around 90 minutes [28]. Figure 2 shows the various stages of sleep and how a person transitions through these stages over the course of a 7 hour sleep.

After sleep is initiated, one enters Stage 1 sleep. In this stage, most cognitive and physiological activities slow down, though body movements still occurs once in a while as sleep travels into a deeper stage. A person wakes up easily from Stage 1 sleep but might recall it as only being drowsy. Stage 2 is seen as an intermediate stage between light and deep sleep, lasting about 10 to 25 minutes. Stage 3 and Stage 4 are considered as deep sleep stages. The person becomes less responsive to external stimuli and the auditory threshold of adults increases compared to Stage 2 and REM sleep [10]. Thus it is relatively harder to awaken a person who is in deep sleep. When awakened from this stage one may feel disoriented for a few minutes. On average, an adult spends 10-20 percent of her sleep time in this slow wave sleep.

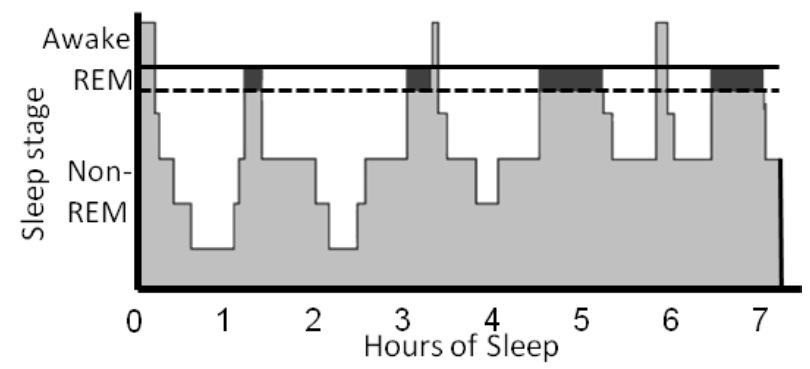

Fig. 2. Sleep architecture: REM sleep and Non-REM sleep and sleep stages. Based on [28].

As a sleep cycle completes and transits to lighter sleep, one enters into REM sleep, also called 'Dream Sleep' [28]. During REM sleep, one's respiration rate and brain activities increase, along with the occurrence of dreams. When awakened in this stage, one is likely to recall his/her dream. But a person is unable to move due to the inability to use his/her voluntary muscles, e.g. postural or skeletal muscles.

\subsection{Sociology of Sleep}

Since as far back as the 16th century there exists literature on the etiquette of sleep and the sharing of a bed. It was not uncommon [56] for medieval households to share a huge bed among all household members including servants and knights. Often only wealthy nobles with large houses could afford separate bedrooms. Sleep in those times was a public affair that required ordering and coordination.

Although sleeping habits have changed and evolved in the 21st century it is still the case that members of a collective coordinate "time schedules" for sleep. For example, the arrival of a new child compromises the sleeping schedule of a couple to the extent that synchronization of the baby's sleep with the parents' becomes an imperative. The sleep synchronization of the youngster not only allows parents to reduce disturbance 
during their sleep but it also hastens the child's integration into the family's pattern of activity. This is not only true of a family but of many other groups. Apartment dwellers in urban environments have noise level restrictions at night to reduce sleep disturbance of others.

The scheduling within a collective generally serves two main purposes: first, when sleeping, it reduces external stimuli that can disturb the sleep of an individual, and second, when awake, it allows members of the collectivity to interact with each other.

Another important sociological aspect of sleep is safety. The sleeping society is extremely vulnerable since it not only lacks the sense of location but also control over its social and physical environment. The collective coordination of sleep potentially minimizes this vulnerability to an extent.

Schwartz [49] argues that the transition from sleep to the wake state is a necessity for protecting a person against sudden shifts or impositions of a wake status, since immediately after waking up a person needs time to gain full control of his or her body and mind. The time needed for the transition will vary depending on a person's mental and physical state, age and other factors. Similarly, bed time rituals play an important role in the transitions from being awake to sleep [49, 57]. Familiar cues, such as the end of a TV program, often set into motion the bedtime ritual. These rituals (such as undressing, washing or donning the sleeping costume) are not only a response to sleepiness but they are also triggers to induce sleep [49].

Another aspect of sleep is how the presence of the sleeper affects those that are awake. The effect depends on the context and the relation between those sleeping and other wakeful persons e.g. an insomniac being wide awake at night might feel helpless realizing other people are asleep.

There are also different sleeping cultures, which are: monophasic (sleep consolidated into one block), bi-phasic (two blocks of sleep during the day and night), and polyphasic (one night's sleep and several day's napping). These sleeping cultures are often associated with a particular country or region e.g. bi-phasic is common for southern countries like Spain (i.e. siesta), while polyphasic is most common in Japan (i.e. napping called inemuri - "to be present and sleep"). It is important to understand these differences when designing for sleep. For example based on the physiology of sleep napping has a positive effect on the well-being of a person, however social norms might not accept napping at work.

Other cross-cultural differences regarding sleep also include bedtime rituals, cosleeping (of infants with parents), the sleeping environment and sleep related problems [38]. For example co-sleeping with young children in Japan (59\%) was found to be much higher than in US (15\%) [38]. As a result, a product intended for supporting healthy bedtime routines for US families may not work well for Japanese families and vice versa.

Many sociological aspects of sleep are dictated by sleep physiology and how the body regulates its sleep and wake cycles. Understanding the physiology of sleep is also essential for designing supporting technologies.

\subsection{Psychological Aspect of Sleep}

Many sleep problems are caused by psychological factors, such as worries, a busy mind, stress and anxiety $[4,15]$. According to the survey from the National Sleep 
Foundation [36], 35\% of respondents reported that worries and not being able to empty one's mind are the most disturbing psychological factors affecting sleep. Psychological responses, such as stress and anxiety correspond to physiological changes like increased respiration rate, heart rate, decreased skin temperature and increased body temperature [6]. These physiological changes enhance alertness and arousal and are contrary to the decreased physiological state of a person falling asleep. Studies that compare good and poor sleepers provided further evidence that poor sleepers have increased anxiety and alertness at bedtime, resulting in decreased skin temperature [26], increased number of body movements, and increased core body temperature [32].

Therefore, many sleep enhancing products are designed based on meditation principles [51], such as muscle and mind relaxation and breathing techniques to induce relaxation. These products have the potential to reduce sleep onset when used at bedtime, such as the paced breathing devices of emWave ${ }^{\odot}$ (www.emwaveforsleep.com) and nightWave ${ }^{\circledR}$ (www.nightwave.com).

\subsection{Sleep Problems and Coping Strategies}

According to the American Academy of Sleep Medicine, there are various types of sleep disorders, ranging from respiratory-related sleep disorders to developmental or neuropsychiatric sleep disorders. Some of these disorders have a medical cause, while others are related to behavioral or cognitive factors. The latter implies that the treatment should focus on the behavioral and / or cognitive basis of these conditions. The term commonly used for these type of treatments is Cognitive Behavioral Therapy (CBT-I). It is a treatment for insomnia, since misperceptions regarding sleep and stressful events often play an important role in the development of insomnia. For example, people might have problems falling asleep and therefore become more and more anxious as the night passes. As a result they might engage in behaviors that are detrimental for sleep, such as working behind the $\mathrm{pc}$ in the bedroom. The aim of CBT-I is to break such patterns by providing insight into people's actual sleeping behavior and educating them to associate the bedroom with sleeping. Review studies have shown the effectiveness of such treatments, reflected by a significant reduction in sleeping problems, which exceed those of pharmacological treatments [52] and last longer [45].

\subsection{Social and Demographic Factors Influencing Sleep}

The physiology as well as sociology of sleep define how the different external (environmental) and internal factors influence sleep and sleep quality. Factors, such as location, the people around us and our age can have a direct effect on the way we prepare ourselves for sleep, how we cope with sleepiness, and how we wake up. In this section we describe factors that we believe have the biggest influence on sleep and therefore must be considered during designing sleep related interaction technology.

Location. Our ability to fall asleep and the quality of sleep heavily depends on where we sleep. An individual can sleep well at home while suffering from insomnia in a hotel room. 
Factors such as unfamiliar noises [17, 35], additional stress (as in a hospital) [13], or an uncomfortable bed can all influence our ability to sleep. Alongside the unfamiliarity of a location, comes our anticipation of safety [49, 56]. For example being able to sleep for half an hour in a train may refresh us but the anxiety about safety while sleeping will make it very difficult to fall asleep. People living in warzones or in refugee camps often suffer from sleep related disorders due to anxiety. Getting a good night's sleep has been found to be difficult for the 62 million people living in relatively unsafe refugee camps.

Another aspect that affects our sleep is the social expectations and norms of the location. The way we can make ourselves comfortable (say undressing or curling up) at home in our bed may not be socially acceptable in an airline lounge even if they have a flat bed. In general, understanding what effect the location has on sleep can trigger the creation of new supportive interaction technologies.

The People around Us. Another important contextual factor is the people who are around us when we are about to sleep. For example, families without children have very different sleeping routines, sleep duration and problems when compared to families with children or to people living alone $[3,18]$.

Partners sharing one bed face disturbances such as, snoring, individual differences in room temperature preferences and different bedtime habits (wanting to read before sleeping or watching TV). In families with children, pre-sleep as well as waking-up routines are important activities for ensuring good sleep for both the children and the parents. There maybe other routines in place for when the parent wakes up due to young child having difficulty falling asleep. Interestingly partners often say they have a better sleep when they are together instead of sleeping alone, while objective measurements show the opposite.

Recently, a number of different concepts for re-connecting couples and family members have been proposed $[9,11,29,30]$. These concepts could indirectly help to improve sleep quality by creating 'peace of mind'. However they can also be extended to include sleep as an essential part of re-connecting by creating a more intimate link between partners.

Age. People of different ages sleep differently, have different routines and experience different problems with sleep [5, 21]. For example, elderly people suffer from insomnia more often and overall have a lower quality of sleep. Their sleep tends to be more fragmented and their waking up time is earlier than young adults [28]. Elderly people tend to undertake fewer physical activities, have less light exposure and often nap during the day; these factors hamper their night's sleep. Consequently, new solutions should help them to manage a healthier and more physically active routine, not just before sleep but throughout the day. In contrast, busy adults, are often more stressed which, despite generally being in good health, might lead to problems with falling asleep. Solutions for stress management, relaxation guidance and enabling power naps could be suitable for this target group. Sleep enhancing solutions should therefore be designed differently for the two age groups.

\section{Opportunities for Design}

Thus far, we have discussed how sleep is a dynamic process and affected by various external and internal factors. When designing products or services that support the 
ritual or activities that influence sleep, we should take into account its basic physiological, psychological and sociological characteristics, as well as contextual factors such as location and culture. Below we propose design opportunities that relate to the aforementioned four factors.

The opportunities are structured around five themes: scheduling for the collective; sleep management; supporting transitions; context of and around sleep; and feigning and inhibiting sleep.

\subsection{Scheduling for a Collective}

As we noted in the section on Sociology of Sleep, scheduling for a collective is an important sociological aspect of sleep. Traditionally the ordering and time coordination of sleep has been a loosely scheduled activity. But with busy lifestyles and more distributed collective, scheduling sleep these days becomes a more complex activity that can benefit from careful technology mediated design. Below we list some examples of problems with coordination and potential opportunities for HCI.

Although the authors of the reverse alarm clock [39] and the networked alarm clock [48] do not discuss their designs from the perspective of scheduling for a collective, both these designs are examples that promote coordination between members of a collective. In the first instance the alarm clock is encouraging the child to coordinate his awakening to that of his parents.

However, with the latest developments in sleep sensing technologies (such as Zeo [58] described in the previous section) it is possible to redesign the coordination strategies through a much richer user experience. For example, the reverse alarm clock's design can be extended to include children in the age group of $4-7$ yrs by incorporating appropriately presented information about their parents' sleep state. This could encourage children to only awaken their parents when it's absolutely essential or if they are in a sleep state that will allow them to respond immediately upon being awakened (which can be detected by monitoring the sleep stage of a person). Such awareness of a sleeping member is valuable for all members of a household (not just children). For example if the door of an elderly person living in cared facility could provide awareness cues of the sleep state of the person (information like - when they fell asleep and when are they likely to wake-up) it might help care-givers to decide whether to return later or wake them up. Another usage of sleep awareness was proposed in the Aurama system [9]. The Aurama is a context aware system that provides caregivers with information about elders living independently [9]. The load sensors in the bed were used to distinguish between, moving and staying still (i.e. wake and sleep states); this information together with overall sleep patterns was available to the caregiver. However detailed information about a sleep state, such us lying in bed awake, falling asleep or sleeping, was not available. Such awareness possibilities are only possible now with the relatively robust single electrode EEG sets like Zeo [58].

This form of awareness information can also be shared via network enabled devices so a distributed family can coordinate and schedule their activities. There are interesting design challenges in supporting such activities for a distributed collective that is spread across multiple time-zones. Family calendars and devices could include asleep or in bed mode to let remote participants know that they are not available. 
When shared within a social network, it may help strengthen the social relationships between friends and therefore may add a new dimension to the current social relationships in groups.

Such collective scheduling of sleep could also be used by people to plan activities around time when others might be asleep (in a way "abuse" sleep time). For example, one may choose to set-off on a long journey at night to avoid traffic or use the quiet time to work or play on their media console. In these cases a person might not only choose to not disclose their sleep related awareness information but it is also possible to use the relevant networked information to plan their activity to reduce their vulnerability (can I get a head-start on the local traffic?) or capitalize on others vulnerability (to carry out anti-social behavior). This will need to be taken into account when designing a system for supporting sleep awareness for families as well as for social networks.

\subsection{Sleep Management}

Sleep management refers to how we organize our sleep. As we have described in the sleep section many sleep problems come from psychological disturbances, inappropriate sleep routines or busy lifestyles. As a result people start to sleep less [28]. In this situation being able to get a short nap during the day is not anymore a luxury but a necessity. Design solutions aimed at sleep management can therefore not only address night time sleep but also daytime naps. Below we present two examples of designing systems for improving sleep management.

Event Driven Alarms. Most HCI work related to sleep has revolved around the redesign of the alarm clock. The main focus of the redesign lies in adjusting the wake up time based on predetermined condition(s) and in providing contextual information that would help to adjust the usual morning routine if necessary. Despite adding context and events the proposed alarm clock modifications are still time based. While these are useful in a bedroom setting these alarms are of relatively little use in locations like the office or train. It might be interesting to explore alarms that can awaken a person based on a specific event without including any time constraints.

Although the network alarm clock [48] is an event driven alarm clock where the event is what other members of the social network of the person are doing, the authors do not interpret their alarm clock as an event-driven clock and potentially miss some interesting design possibilities that emerge from this form of interpretation which leads to more possible forms of sleep management. For example, a person napping in a train would find it useful if the clock went-off when their station is approaching. Rather than having a time-based alarm, it would go off at the anticipated station arrival time. The clock could monitor the train information and the user's sleep stage to trigger the alarm by optimizing arrival time with sleep stage to wake the person at the appropriate moment. Similarly a project coordinator might choose to nap until he receives a critical email from a colleague. An event driven alarm could help the person have a more "intense" nap knowing fully well that they will be awakened by the alarm only when an email from the right person arrives. This can lead to well rested employees and improved sleep scheduling through napping. 
Improving Sleep Hygiene. Opportunities exist to combine remote day time activity monitoring and sleep monitoring technologies to draw a complete picture of day time and night time behavior. As discussed previously, sleep is influenced by many psycho-physiological factors, such as light exposure, physical activity, drinking and eating behavior throughout a day. Thus, more tailored advice can be provided to individuals as a persuasive tool to improve their sleep behavior.

\subsection{Supporting Transitions}

Supporting transition phases of sleep (sleep onset and waking up) can help a person fall asleep and wake up feeling fully rested. One needs to carefully consider the physiological and psychological aspects of falling asleep and waking up.

The main challenge during winding down is to reduce the disturbances from the environment by supporting consistent bedtime routines by adapting familiar routines for different location and people around us. For example, bedtime routines from a bedroom can be adapted and re-appropriated for an airline lounge or office nap. Similarly, routines can be adapted for minimal disruption when a bed partner is away on business trip.

Recently several exciting products that support waking up such as EasyWakeup ${ }^{\circledR}$ (easywakeup.net), and Philips Wake-up Light ${ }^{\odot}$, have become available. Making the waking up more gradual (e.g. such as with the Philips Wake-up Light) can insure a pleasant wake up experience. However these products rarely consider supporting different requirements of couples who have different wake-up schedules. The additional challenge here is to achieve a gradual wake up without disturbing the sleeping partner.

Another example is expressive alarm clock described in [55], where the alarm sound of the clock depends on the way the user sets the alarm time (emotional state and level of urgency). As described in the sleep section the waking moment as well as how a person was awakened affects how the person feels. By using sleep monitoring device to detect the sleep stage the waking up experience can be further enhanced by alarm sound selection and by shifting the actual wake up moment (e.g. based on the level of urgency).

Sleep Onset in the Presence of Multi-media. Nowadays it is increasingly common for the bedroom to include a television and for bedtime rituals to include watching TV in bed. Watching TV in bed is generally considered a bad influence on sleep. The constantly changing multimedia images and evolving storylines in the program can cause anxiety and increase arousal, whereas to induce sleep onset one needs to be relaxed and calmed. In addition, the bedroom should be associated only with sleep [53]), thus watching TV should be kept outside the bedroom. While moving the TV out of the bedroom is a simple solution it is unfortunately not the desired solution for most people and that is one reason why every hotel room today includes a TV in the bedroom.

The challenge here is to redesign the TV such that it facilitates the transition from awake to sleep for the user. For example, the TV could have a "bedtime" mode where the TV recommends a program that not only includes appropriate content but also includes visual elements that avoids blue, or slowly reduces the contrast, brightness, 
volume and image quality to create a blurry 'sleepy' effect, that can help to induce sleep onset.

When a user transits from being awake to asleep his/her capabilities as well as needs for interaction changes. For example, when a person is almost asleep he only needs an "off" button whilst when awake a "change channel" button is more appropriate. The decreasing of cognitive and physical state of a person during a sleep initiating process means a bedroom product should adapt and alter its interaction mode from explicit (requiring active user control) to implicit (fully automatic). When a user is fully awake, he/she should be able to take full control over the device, whereas, when he/she is fully asleep, the device should operate automatically. The challenge lies in balancing the two types of interactions for the transition zone from explicit to implicit and on the degree of control the system has. Users do not always value systems that think for them, even when they are not in a state to do so themselves.

At bedtime, bedrooms are quiet and the light level is generally lower, with people avoiding too much physical activity (except for sex, [53]). Therefore, an interface that requires precise user input, e.g. small buttons, or has a light based user interface, e.g. LCD screen, are not suitable in this context. In such situations UIs need to be redesigned to allow quick and effortless interaction; effortless meaning minimal rise in a user's arousal.

\subsection{Context of and around Sleep}

People sleep in different contexts which can also affect their quality of sleep, for example, one might require a dark and quiet room to sleep in at night, but one may be less conscious about sleeping on a noisy and crowded train. People might find it disturbing when sharing a bed with a partner but at the same time feel lonely when alone in bed. These different contextual requirements require careful consideration when designing for and around sleep. Below we describe one such context in further detail.

Bed Sharing Context. People who sleep by sharing a bed with a partner need to face and deal with issues such as different bedtime schedules, rituals and preferences regarding the sleeping environment. Therefore knowing the preferences of each individual in the bed and adjusting the context or setting of devices in the bedroom can help reduce conflict. A bedroom TV could include a "partner asleep" mode where the volume and brightness settings are adjusted or the TV adapts its orientation (either by physically turning or by using the emerging multi-view 3D displays) to minimize light for the sleeping partner. Waking up alarms can also be designed to use different actuation modes, such as, vibration when one partner wants to sleep for longer, or audio alarms when both partners want to get up at the same time. These forms of contextual design are becoming important as couples have increasingly different work schedules and demands.

\subsection{Feigning and Inhibiting Sleep}

Most of our discussion up to this point has been on improving the quality of sleep and the experiences around sleep. However there are several situations where it may be 
useful to feign or inhibit sleep. For example, yawning (real or not) is an important social marker; it indicates to the collective that a person is tired. It may be rude in some settings but in others, it offers the perfect opportunity to excuse oneself from a gathering; a yawn at a dinner party can be followed by a remark about how tired one feels and the request to be excused. It is also not uncommon to feign sleep to listen in on others' conversations or to avoid conversations with a bed mate.

There are opportunities to explore how systems can be designed to support such social activities. If sleep monitoring technology and awareness tools become prevalent and popular, it is possible to envisage situations where the user reappropriates the awareness provided by these systems to feign sleep or tiredness. There are bound to be ethical debates on how far one can go with such reappropriation.

An important aspect of sleep management is inhibition of sleep. Stimulants such as coffee are often used to inhibit sleep but their effectiveness has always been questioned depending on the amount of actually consumed caffeine.

Minimizing the need for sleep has been the holy grail for war planners since time immemorial [33]. American pilots are often given Amphetamines (otherwise known as speed) to keep them alert for 30-hour long missions. There are several military research programs (from US and other governments) in place to explore 90-hour long shifts.

Such research ventures are not only of interest to the military but also to shiftworkers and increasingly for professionals such as stock analysts, surgeons etc. Most legal sleep inhibition approaches in office environments today rely on stimulants, such as caffeine present in coffee and RedBull ${ }^{\odot}$. New ways of sleep inhibition through minimal use of stimulants is of great interest to firms that deal in time-critical work.

Design solutions that combine persuasion techniques with a better understanding of the physiology and sociology of sleep can lead to systems that inhibit sleep. Such solutions can explore passive approaches such as adjusting the lighting condition, bringing together people who need to inhibit sleep and so on. While these approaches can work they may be too slow for a rapidly evolving situation. In such cases, design solutions can also explore electro-magnetic stimulation to temporarily suppress sleep receptors [33]. Such approaches need to be carefully debated and considered from a legal and ethical standpoint.

\subsection{Summary}

The following list summarizes opportunities identified in each subsection:

- Supporting sleep awareness within a family (co-located and distributed) or a community (students living in a dorm or cared living for older people) and social network aided by sleep sensing technologies

- Supporting event driven wake-up, where event include external factors (e.g. traffic, weather, destination) and internal factor (e.g. sleep stage, desired wake up time, urgency, partner sleep stage, specific wake-up triggers defined by the user)

- Using persuasive tools to provide tailored advice for improving sleep hygiene. 
- Supporting sleep onset in the presence of multi-media distracters such as TVs. For example, the color and tone-mapping in these devices can be carefully adjusted through detailed understanding of retinal response and color-perception theory to positively influence the cycle.

- Context sensitive intervention that support sleep for an individual while accommodating potentially conflicting needs of the partner. For example, preserving an individual's sleep ritual of reading a book in the bed while simultaneously being sensitive to the needs of her partner to sleep immediately.

- Persuasive tools, techniques and systems to facilitate sleep inhibition in different situations and contexts.

\section{Conclusions}

The main contributions of the paper lie in the exploration of a newly emerging topic in the area of sleep that is becoming more important due to changes in society and lifestyle and presents challenges for which the HCI community can rise to. Through a review of the literature we describe the phenomenon of sleep and its position in the social life to provide a rich understanding of Sleep. We present a set of factors that have a direct effect on the way we prepare ourselves for sleep, cope with sleepiness, or wake-up and identify five sleep related themes that have high potential and need for interaction design.

We believe this paper opens up a new topic for HCI research and will serve as a beneficial reference for future researchers.

\section{References}

1. Ancoli-Israel, S., Cole, R., Alessi, C., Chambers, M., Moorcoft, W., Pollak, C.P.: The role of actigraphy in the study of sleep and circadian rhythms. American Academy of Sleep Medicine Review Paper. Sleep 26, 342-392 (2003)

2. Balkin, T.J., Braun, A.R., Wesensten, N.J., Jeffries, K., Varga, M., Baldwin, P., Belenky, G., Herscovitch, P.: The process of awakening: a PET study of regional brain activity patterns mediating the reestablishment of alertness and consciousness. Brain, 2308-2319 (2001)

3. Biddle, J.E., Hamermesh, D.S.: Sleep \& the allocation of time. The Journal of political economy 98(5-1), 922-943 (1990)

4. Bertelson, A.D., Monroe, L.J.: Personality patterns of adolescent poor and good sleepers. Journal of Abnormal Child Psychology 7, 191-197 (1979)

5. Bovill, M., Livingstone, S.: Bedroom culture and the privatization of media use. In: Bovill, M., Livingstone, S. (eds.) Children \& their changing media environment: a European comparative study. Lawrence Erlbaum, Mahwah (2001)

6. Cannon, W.B.: Bodily changes in pain, hunger, fear and rage: An account of recent research into the function of emotional excitement, 2nd edn. Appleton-Century-Crofts, New York (1929)

7. Chesson, A.L., Ferber, R.A., Fry, J.M., Grigg-Damberger, M., Hartse, K.M., Hurwitz, T.D., Johnson, S., Kader, G.A., Littner, M., Rosen, G., Sangal, R.B., Schmidt-Nowaram, W., Sher, A.: The indications for polysomnography and related procedures. Sleep 20, 423-487 (1997) 
8. Choe, E.K., Kientz, J.A., Halko, S., Fonville, A., Sakaguchi, D., Watson, N.F.: Opportunities for computing to support healthy sleep behavior. In: Ext. Abstracts CHI 2010, pp. 3661-3666. ACM, New York (2010)

9. Dadlani, P., Sinitsyn, A., Fontijn, W., Markopoulos, P.: Aurama: caregiver awareness for living independently with an augmented picture frame display. AI \& Society 25(2), 233-245 (2010)

10. Davis, H., Davis, P.A., Loomis, A.L., Harvey, E.N., Hobart, G.: Electrical reactions of the human brain to auditory stimulation during sleep. Journal of Neurophysiology 2, 500-514 (1939)

11. Dodge, C.: The bed: a medium for intimate communication. In: Ext. Abstr. CHI 1997. ACM Press, New York (1997)

12. Edinger, J.D., Fins, A.I., Sullivan, R.J., Marsh, G.R., Dailey, D.S., Hope, T.V., Young, M., Shaw, E., Carlson, D., Vasilas, D.: Sleep in the laboratory and sleep at home: comparisons of older insomniacs and normal sleepers. Sleep 20, 1119-1126 (1997)

13. Freedman, N., Kotzer, N., Schwab, R.: Patient perception of sleep quality and etiology of sleep disruption in the intensive care unit. Am. J. Respir. Crit. Care. Med. 159, 1155-1162 (1999)

14. Freedman, R.R.: EEG power spectra in sleep-onset insomnia. Electroencephalography and Clinical Neurophysiology 63(5), 408-413 (1986)

15. Fortunato, V., Harsh, J.: Stress \& Sleep Quality: The Moderating Role of Negative Affectivity. Personality and Individual Differences 41, 821-836 (2006)

16. Foulkes, D., Vogel, G.: Mental activity at sleep onset. Journal of Abnormal Psychology 70(4), 231-243 (1965)

17. Griefahn, B., Spreng, M.: Disturbed sleep patterns and limitation of noise. Noise \& Health 6(22), 27-33 (2004)

18. Hale, L.: Who has time to sleep? Journal of Public Health 27(2), 205-211 (2005)

19. Hauri, P.J., Wisbey, J.: Wrist actigraphy in insomnia. Sleep 15, 293-301 (1992)

20. Hirshkowitz, M., Moore, A.C., Hamilton, C.R., Rando, K.C., Karacan, I.: Sleep apnea in patients with quadriplegia. Journal of Clinical Neurophysiology, 56-62 (1992)

21. Hume, K., Van, F., Watson, A.: A field study of age \& gender differences in habitual adult sleep. Journal of Sleep Research 7(2), 85-94 (1998)

22. Jewett, M.E., Kronauer, R.E., Czeisler, C.A.: Phase/amplitude resetting of the human circadian pacemaker via bright light: A further analysis. Journal of Biological Rhythms 9, 295-314 (1994)

23. Kalra, G.K., Banks, S., Dinges, D.F.: Relationship between polysomnography and simultaneous actigraphy in healthy subjects. Poster Presented at Sleep, 22nd Annual Meeting of the Associated Professional Sleep Societies, LLC (2008)

24. Kim, S., Kientz, J.A., Patel, S.N., Abowd, G.D.: Are you sleeping?: sharing portrayed sleeping status within a social network. In: Proc. CHI 2008, pp. 619-628 (2008)

25. Krauchi, K., Cajochen, C., Wirz-Justice, A.: Waking up properly: is there a role of thermoregulation in sleep inertia? Journal of sleep research, 121-127 (2004)

26. Lack, L.C., Gradisar, M., Van Someren, E.J.W., Wright, H.R., Lushington, K.: The relationship between insomnia and body temperatures. Sleep Medicine Reviews 12(4), 307-317 (2008)

27. Landry, B.M., Pierce, J.S., Isbell, C.L.: Supporting routine decision-making with a nextgeneration alarm clock. Per.Ubiq. Comput. 8(3-4), 154-160 (2004)

28. Lawrence, L.J., Mardon, S.: The Harvard Medical School guide to a good night's sleep. McGraw-Hill, New York (2007) 
29. Lottridge, D., Masson, N., Mackay, W.: Sharing empty moments: design for remote couples. In: Ext. Abstracts CHI 2009, pp. 2329-2338. ACM, New York (2009)

30. Mhorain, A., Agamanolis, S.: Aura: an intimate remote awareness system based on sleep patterns. In: Proc. of the CHI Workshop on Awareness Systems (2005)

31. Miltler, M.M., Miller, J.C.: Methods of testing for sleepiness. Behavioral Medicine 21(4), 171-183 (1996)

32. Monroe, L.J.: Psychological and physiological differences between good and poor sleepers. Journal of Abnormal Psychology 72, 255-264 (1967)

33. Moreno, J.: Juicing the brain. Scientific American Mind 17(6), 66-73 (2006)

34. Morin, C.M.: Insomnia: Psychological Assessment and Management. Guilford, New York (2003)

35. Muzet, A.: Environmental noise, sleep and health. Sleep Medicine Reviews 11, 135-142 (2007)

36. National Sleep Foundation. 2008\&2010 Sleep in America Poll, http://www.sleepfoundation.org

37. Ogilvie, R., Wilkinson, R.: The detection of sleep onset: behavioral and physiological convergence. Psychophysiology 21, 510-520 (1984)

38. Owens, J.A.: Sleep in children: Cross-cultural perspectives. Sleep and Biol. R. 2, 165-173 (2004)

39. Ozenc, K.F., Brommer, J.P., Jeong, B.-k., Shih, N., Au, K., Zimmerman, J.: Reverse alarm clock: a research through design example of designing for the self. In: Proc. DPPI 2007, pp. 392-406. ACM, New York (2007)

40. Patel, S.R., Hu, F.B.: Short sleep duration and weight gain: a systematic review. Obesity (Silver Spring) 16(3), 643-653 (2008)

41. Piggins, H.D.: Human clock genes. Annals of Medicine 34(5), 394-400 (2002)

42. Punjabi, N.M., Shahar, E., Redline, S., Gottlieb, D.J., Givelber, R., Resnick, H.E.: SleepDisordered Breathing, Glucose Intolerance, and Insulin Resistance. American Journal of Epidemiology 160(6), 521-530 (2004)

43. Renger, J.J.: Overview of Experimental and Conventional Pharmacological Approaches in the Treatment of Sleep and Wake Disorders. Current Topics in Medicinal Chemistry 8, 937-953 (2008)

44. Richardson, G., Tate, B.: Hormonal and pharmacological manipulation of the circadian clock: recent developments and future strategies. Sleep 23, 77-85 (2000)

45. Riemann, D., Perlis, M.L.: The treatments of chronic insomnia: a review of benzodiazepine receptor agonists and psychological and behavioral therapies. Sleep Medicine Reviews 13(3), 205-214 (2009)

46. Sadeh, A., Hauri, P.J., Kripke, D.F., Lavie, P.: The role of actigraphy in the evaluation of sleep disorders. Sleep 18, 288-302 (1995)

47. Scherini, T., Melo, P., van Craenendonck, T., Zou, W., Kaptein, M.: Enhancing the sleeping quality of partners living apart. In: Proc. DIS 2010, pp. 171-174. ACM, New York (2010)

48. Schmidt, A.: Network alarm clock. Per.Ubiq. Comput. 10(2-3), 191-192 (2006)

49. Schwartz, B.: Notes on the Sociology of Sleep. The Sociological Quarterly 11(4), 485-499 (1970)

50. Sermon, P.: Telematic Dreaming. Leonardo 33(2) (2000)

51. Shamini, J., Shauna, L.S., Summer, S., Scott, C.R., Paul, J.M., Iris, B., Gary, E.R.S.: A randomized controlled trial of mindfulness meditation versus relaxation training: Effects on distress, positive states of mind, rumination, and distraction. Analysis of behavioral medicine 33(1), 11-21 (2007) 
52. Smith, M.T., Perlis, M.L., Park, A., Smith, M.S., Pennington, J.M., Giles, D.E., Buysse, D.J.: Comparative meta-analysis of pharmacotherapy and behavior therapy for persistent insomnia. American Journal of Psychiatry, 5-11 (2002)

53. Stepanski, E.J., Wyatt, J.K.: Use of sleep hygiene in the treatment of insomnia. Sleep Medicine Reviews 7(3), 215-225 (2003)

54. Verkasalo, P.K., Lillberg, K., Stevens, R.G., Hublin, C., Partinen, M., Koskenvuo, M., Kaprio, J.: Sleep Duration and Breast Cancer: A Prospective Cohort Study. Cancer Research 65(20), 9595-9600 (2005)

55. Wensveen, S.A.G., Overbeeke, C.J., Djajadiningrat, J.P.: Push me, shove me and I show you how you feel. Recognizing mood from emotionally rich interaction. In: Proc. of DIS 2002, London, UK, June 25-28, pp. 335-340 (2002)

56. Williams, S.J.: The Social Etiquette of Sleep: Some Sociological Reflections and Observations. Sociology 41(2), 313-328 (2007)

57. Williams, S.J.: The Sociological Significance of Sleep: Progress, Problems and Prospects. Sociology Compass 2(2), 639-653 (2008)

58. Zeo Personal Sleep Coach, http: //www. myzeo.com/ 